\title{
Lifshitz point in the phase diagram of a ferroelectric liquid crystal in an external magnetic field
}

\author{
M. Škarabot, R. Blinc, and I. Muševic \\ J. Stefan Institute, Jamova 39, 1000 Ljubljana, Slovenia
}

A. Rastegar and Th. Rasing

Research Institute for Materials and High Field Magnet Laboratory, University of Nijmegen, Toernooiveld, 6525 ED Nijmegen, The Netherlands

(Received 26 October 1999)

\begin{abstract}
The Lifshitz point in the $(H, T)$ phase diagram of a ferroelectric liquid crystal in a transverse magnetic field has been determined using photon correlation spectroscopy and linear electrooptic response measurements. The extrapolated Lifshitz magnetic field is $25(1 \pm 0.1) \mathrm{T}$ for a smectic- $C^{*}$ material with an unperturbed helical period of $\approx 6 \mu \mathrm{m}$, and the Lifshitz point is located $\approx 100 \mathrm{mK}$ above the zero-field transition temperature. We have observed the reentrant helical modulated smectic- $C^{*}$ phase just below the $\lambda$ line. The width of this phase is $100 \mathrm{mK}$ at the low temperature critical field $H_{c}$ and decreases continuously to zero as we approach the Lifshitz point. The phase boundary between the reentrant phase and the smectic- $A$ phase is of second order, whereas the phase boundary with the unwound smectic- $\bar{C}^{*}$ phase is of first order far away from the Lifshitz point and becomes of second order close to the Lifshitz point. The order parameter dynamics is discussed within the Landau theory.
\end{abstract}

PACS number(s): 61.30.-v, 64.70.Md, 64.60.Ht

\section{INTRODUCTION}

The Lifshitz point was first discussed by Hornreich, Luban, and Shtrikman in 1975 [1] and introduced to the field of liquid crystals by Michelson [2]. It is a triple point, where the disordered smectic- $A$, the homogeneously ordered smectic- $\bar{C}^{*}$ and the inhomogeneously ordered smectic- $C^{*}$ phases meet, as shown in Fig. 1.

Michelson proposed the possible physical realization of a Lifshitz point in the $(H, T)$ phase diagram of a ferroelectric liquid crystal, where an external magnetic field is applied perpendicular to the helical axis of the smectic- $C^{*}$ phase. If the diamagnetic anisotropy of the liquid crystalline molecules is positive, molecules tend to align along the field direction. This results in the so-called solitonlike distortion of the helical structure, where long, almost uniformly aligned domains are separated by thin soliton domain walls. At a certain critical field $H_{c}$ the distorted helical structure unwinds into the spatially homogeneous smectic- $\bar{C}^{*}$ structure. Alternatively, the unwound phase can be reached by decreasing the temperature $T$ from the smectic- $A$ phase. For a low external magnetic field, we cross at a certain temperature the phase transition line $T_{\lambda}(H)$ and enter the modulated smectic$C^{*}$ phase. On the other hand, at high enough magnetic fields we cross the phase transition line $T_{0}(H)$ and enter the spatially homogeneous (unwound) smectic- $\bar{C}^{*}$ phase, as shown in Fig. 1. The phase transition lines $T_{\lambda}(H), T_{0}(H)$, and $H_{c}(T)$ thus merge into a Lifshitz point $\left(H_{L}, T_{L}\right)$ where the disordered (smectic- $A$ ), the homogeneously ordered (smectic- $\bar{C}^{*}$ ), and the helicoidally ordered (smectic- $C^{*}$ ) phases meet.

The $(H, T)$ phase diagram of a ferroelectric liquid crystal in an external field was reported in high-magnetic-field experiments on $p$-decyloxybenzilidene- $p^{\prime}$-amino-2-methylbutyl cinnamate (DOBAMBC) by Muševic et al. [3]. The phase boundaries were determined by static light scattering and dielectric spectroscopy and were in qualitative agreement with theoretical predictions. The smectic- $A$-smectic$C^{*}$ phase transition line was found to be practically field independent within the resolution of $20 \mathrm{mk}$ up to $10 \mathrm{~T}$, which is in agreement with the expected increase of the $\lambda$ line. Later on, the experimental work on the $(H, T)$ phase diagram was continued by Seppen [4]. Recently, related experiments were performed in a high-frequency electric field (Wang, Sun, and Feng [5]) which couples quadratically to the dielectric anisotropy and therefore results in an analogous phase diagram. Their results were similar to those reported by Muševič et al. [3].

The magnetic field experiment in chiral DOBAMBC, however, revealed the existence of a reentrant smectic- $C^{*}$ phase very near the $\lambda$ line and indicated a "runaway" of the Lifshitz point in chiral DOBAMBC. Due to a poor signal-tonoise ratio and the very narrow temperature region of the reentrant modulated ferroelectric phase $(\approx 100 \mathrm{mK})$, the run-

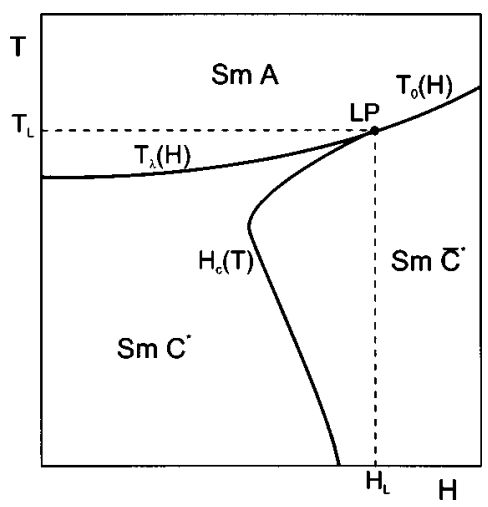

FIG. 1. Schematic view of the $(H, T)$ phase diagram of a ferroelectric liquid crystal in an external magnetic field applied perpendicularly to the helix. Here LP denotes the Lifshitz point $\left(H_{L}, T_{L}\right)$. 
away of the Lifshitz point could not be followed beyond 10 $\mathrm{T}$, so the question of its existence remained open. Recently, the dynamics of ferroelectric liquid crystals in external magnetic fields was studied using quasielastic light scattering and linear response techniques [6]. The experiments were performed far below the $\lambda$ line and reported the observation of magnetic-field-induced band gaps in the dispersion relation for phase excitations propagating along the helical axis. The experiment also clearly proved the advantage of measuring dynamic properties of liquid crystals when determining critical phenomena and phase diagrams. One of the important results of that study was the manifestation of low-frequency, symmetry restoring modes inside the modulated phase. As these modes are slow, their intensities are expected to be large even in the very vicinity of the phase transition. This has reopened the question of the existence and observability of the Lifshitz point in the $(H, T)$ phase diagram of a ferroelectric liquid crystal [7].

In this study we have used both quasielastic light scattering and linear electrooptical response techniques to determine the $(H, T)$ phase diagram of a ferroelectric liquid crystal in an external field. We have used a mixture of purely chiral $(35 \%)$ and racemic liquid crystal 4-(2'-methyl-butyl)phenyl 4'-n-octylbiphenyl-4-carboxylate (CE-8), which has a critical magnetic field $H_{c}=8 \mathrm{~T}$ at $T_{c}-T=3 \mathrm{~K}$ in the ferroelectric smectic- $C^{*}$ phase [8]. We have observed that the critical magnetic field for the unwinding of the helix increases rapidly as we approach the $\lambda$ line, thus forming a very narrow region of a reentrant, magnetically deformed smectic- $C^{*}$ phase. The width of this reentrant phase is of the order of $\approx 100 \mathrm{mK}$ and decreases to zero as we approach the Lifshitz point, which is at $25 \mathrm{~T}$ in this material. Whereas the $\lambda$ line is of second order, the line of the critical magnetic field $H_{c}(T)$ is of second order far below the $\lambda$ line, and becomes of first order near the reentrant phase, and becomes again of second order as it approaches tangentially the $\lambda$ line and the Lifshitz point. This clearly indicates that the observed triple point in the $(H, T)$ phase diagram is indeed a Lifshitz point. The observed order parameter dynamics is discussed within the Landau theory of second order phase transitions.

\section{THEORY}

\section{A. Static properties of the $(H, T)$ phase diagram}

The basic features of the $(H, T)$ phase diagram of a ferroelectric liquid crystal in an external magnetic field can be derived from a simple Landau free-energy expansion

$$
\begin{aligned}
g(z)= & g_{A}+\frac{1}{2} a(T)\left(\xi_{x}^{2}+\xi_{y}^{2}\right)+\frac{1}{4} b\left(\xi_{x}^{2}+\xi_{y}^{2}\right)^{2} \\
& -\Lambda\left(\xi_{x} \frac{\partial \xi_{y}}{\partial z}-\xi_{y} \frac{\partial \xi_{x}}{\partial z}\right)+\frac{1}{2} K_{33}\left[\left(\frac{\partial \xi_{x}}{\partial z}\right)^{2}+\left(\frac{\partial \xi_{y}}{\partial z}\right)^{2}\right] \\
& -\frac{1}{2} \Delta \chi H^{2} \xi_{y}^{2} .
\end{aligned}
$$

Here $g_{A}$ is the equilibrium free energy of the smectic- $A$ phase, $\Delta \chi=\chi_{\|}-\chi_{\perp}$ is the diamagnetic anisotropy, which is assumed to be positive, and the magnetic field is applied in the $y$ direction, $\vec{H}=(0, H, 0)$. We assume that $a(T)=\alpha(T$ $\left.-T_{R}^{*}\right), \alpha$ and $b$ are positive constants, and $T_{R}^{*}$ is the phase transition temperature for the racemic mixture. In Eq. (1), $\Lambda$ is the coefficient of the Lifshitz term and $K_{33}$ is the torsional elastic constant. We assume that the system is homogeneous in the $x-y$ plane, which is parallel to the smectic planes.

The phase transition boundaries between the smectic- $A$ phase and the modulated smectic- $C^{*}$ or homogeneous smectic- $\bar{C}^{*}$ phases can be obtained by the linear stability analysis [2]. As a result, one obtains the so-called Lifshitz field $H_{L}$

$$
H_{L}=\frac{2 \Lambda}{\sqrt{K_{33}|\Delta \chi|}}
$$

which characterizes the phase diagram and determines the position of the Lifshitz point in the $(H, T)$ phase diagram. Below the Lifshitz field $H_{L}$, the smectic- $A$ and the modulated smectic- $C^{*}$ phases are separated by the $\lambda$ line, $T_{\lambda}(H)$,

$$
H<H_{L}, \quad T_{\lambda}(H)=T_{R}^{*}+\Delta T\left[1+\frac{\Delta \chi}{|\Delta \chi|}\left(\frac{H^{2}}{H_{L}^{2}}\right)\right]^{2} .
$$

Here $\Delta T=\Lambda^{2} / \alpha K_{33}$ is the coefficient of the magnetic-field dependence of the $\lambda$ line. As one can see from the equation above, this line increases with the field for positive $\Delta \chi$ and the phase transition is of second order. Above the Lifshitz field the smectic- $A$ phase transforms into the homogeneous smectic- $\bar{C}^{*}$ phase at the second order phase boundary $T_{0}(H)$,

$$
H>H_{L}, \quad T_{0}(H)=T_{R}^{*}+2 \Delta T\left(1+\frac{\Delta \chi}{|\Delta \chi|}\right) \frac{H^{2}}{H_{L}^{2}} .
$$

Beyond the Lifshitz point, the phase transition line increases quadratically with the field.

The most interesting feature is the position of the Lifshitz point $\left(H_{L}, T_{L}\right)$ in the phase diagram. At the Lifshitz point the two phase boundaries $T_{\lambda}(H)$ and $T_{0}(H)$ merge tangentially into each other and to the line of the critical magnetic field $H_{c}(T)$, which separates the modulated and the unwound phases [2]. Near the Lifshitz point, the line of the critical magnetic field $H_{c}(T)$ is of first order [2].

Along the $\lambda$ line, the wave vector $q_{c}(H)$ of the modulated smectic- $C^{*}$ phase decreases smoothly with the field

$$
H<H_{L}, \quad q_{c}(H)=q_{c}(0) \sqrt{1-\left(H / H_{L}\right)^{4}}
$$

from the value $q_{c}(0)=\Lambda / K_{33}$ at $H=0$ to a zero value at and above the Lifshitz field.

The line of the critical field $H_{c}(T)$, which originates in the Lifshitz point and separates the modulated smectic- $C^{*}$ and the homogeneous smectic- $\bar{C}^{*}$ phases, was first calculated numerically by Benguigui and Jacobs [9]. Analytically, it can be derived only in the so-called constant amplitude approximation (CAA). Within the CAA one assumes that the magnitude of the tilt angle does not change significantly with the external field, whereas the phase profile $\Phi(z)$ is allowed to vary with the field. This is a reasonable approximation except within $10 \mathrm{mK}$ around the Lifshitz point [10].

The line of the critical field $H_{c}$ is calculated in the CAA approximation by considering the phase-dependent part of the free-energy density 


$$
\begin{aligned}
g(z)= & -\Lambda \theta^{2} \frac{\partial \Phi(z)}{\partial z}+\frac{1}{2} K_{33} \theta^{2}\left(\frac{\partial \Phi(z)}{\partial z}\right)^{2} \\
& -\frac{1}{2} \Delta \chi H^{2} \theta^{2} \sin ^{2} \Phi(z)
\end{aligned}
$$

After the Euler-Lagrange minimization of the free energy $G=\int g(z) d z$ with respect to $\Phi(z)$ and $\partial \Phi / \partial z$ we obtain the well-known sine-Gordon equation for $\Phi(z)$ [4]. The phase profiles $\Phi(z)$ which minimize the free energy have the form of the so-called $\pi$-soliton lattice and are described by Jacobi's elliptic functions $\sin \Phi_{o}=\operatorname{sn}(u, k)$. Here $\operatorname{sn}(u, k)$ is the Jacobi elliptic sine of the reduced coordinate $u=z /(\xi k)$, where $\xi=\sqrt{K_{33} /\left(|\Delta \chi| H^{2}\right)}$ is the magnetic coherence length. The modulus $k$ of the Jacobi's elliptic function is defined by the equation $k=\left(H / H_{c}\right) E(k)$, where $E(k)$ $=\int_{0}^{\pi / 2} \sqrt{1-k^{2} \sin ^{2} x} d x$ is the complete elliptic integral of the second kind [11]. $H_{c}$ is the critical magnetic field for the unwinding of the helical structure,

$$
H_{c}=\frac{\pi^{2}}{p_{0}} \sqrt{\frac{K_{33}}{\Delta \chi}}
$$

Within the CAA approximation, the critical field is inversely proportional to the period $p_{0}$ of the unperturbed helix, which is similar to the behavior of chiral nematics in an external magnetic field. The critical magnetic field is usually temperature dependent because the period of the unperturbed helix is temperature dependent, too. The phase diagram is "hornlike,' as shown in Fig. 1.

\section{B. Order parameter dynamics for $T \geqslant T_{\lambda}$}

The order parameter dynamics of the smectic- $A$ phase in the presence of a transverse magnetic field is calculated from the free-energy density Eq. (1). The fluctuations are expanded in terms of plane waves with the wave vector along the $z$ direction

$$
\begin{aligned}
& \delta \xi_{x}(z)=\sum_{q} \delta \xi_{q}^{(1)} e^{i q z} \\
& \delta \xi_{y}(z)=\sum_{q} \delta \xi_{q}^{(2)} e^{i q z}
\end{aligned}
$$

The increase of the free energy due to fluctuations, $\Delta G$ $=\int \Delta g(z) d z$, can be written in a matrix form

$$
\Delta G=\vec{\Psi}_{q}^{+} \underline{D}(q) \vec{\Psi}_{q}
$$

Here $\vec{\Psi}_{q}^{+}=\left(\delta \xi_{q}^{(1)}, \delta \xi_{q}^{(2)}\right)$ is the Hermitian conjugate to the fluctuation vector $\vec{\Psi}_{q}$ and the dynamical matrix $\underline{D}(q)$ is

$$
\underline{D}(q)=\left[\begin{array}{cc}
\frac{1}{2} a(T)+\frac{1}{2} K_{3} q^{2} & -i \Lambda q \\
+i \Lambda q & \frac{1}{2} a(T)+\frac{1}{2} K_{3} q^{2}-\frac{1}{2} \Delta \chi H^{2}
\end{array}\right] .
$$

The order parameter dynamics is obtained from the nonequilibrium free energy by applying the Landau-Khalatnikov equations

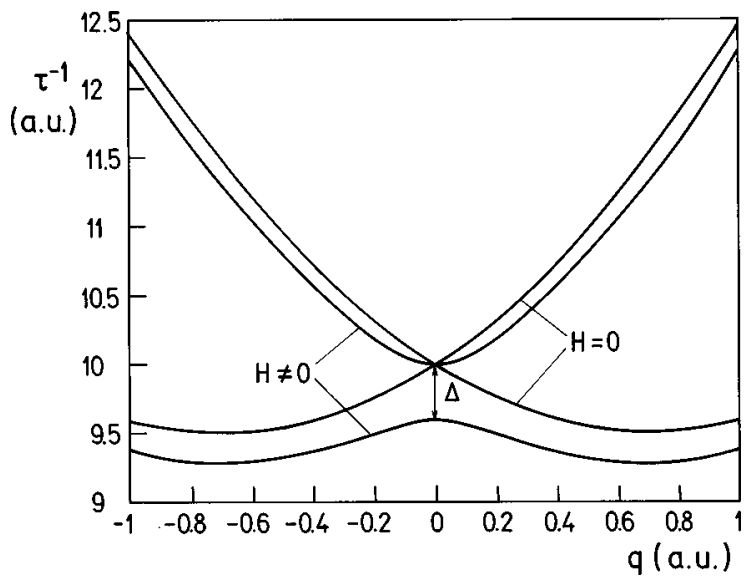

FIG. 2. For zero magnetic field, there are two branches of soft excitations in the smectic- $A$ phase, which are degenerate at $q=0$. For $H \neq 0$, the field induces a band gap $\Delta$ at $q=0$, which removes this degeneracy. The lower branch of soft excitations is critical and condenses at $T_{L}$.

$$
\frac{d \vec{\Psi}_{q}}{d t}=-2 \underline{\Gamma} \underline{\underline{\Psi}} \vec{\Psi}_{q}
$$

Here, the matrix $\Gamma_{i j}=1 / \gamma_{i j}$ is composed of reciprocal viscosity coefficients for the rotational motion of the director. This leads to a set of linear equations which have two nontrivial solutions for each wave vector $q$,

$$
\begin{aligned}
\tau_{+-}^{-1}(q, T)= & \frac{a(T)+K_{3} q^{2}-\frac{1}{2} \Delta \chi H^{2}}{\gamma} \\
& \pm \frac{1}{2 \gamma} \sqrt{\left(\Delta \chi H^{2}\right)^{2}+16 \Lambda^{2} q^{2}}
\end{aligned}
$$

There are two distinct excitation branches in the smectic- $A$ phase in the presence of a transverse magnetic field, which are shown in Fig. 2. The magnetic field induces a splitting of both branches at zero wave vector, $q=0$. This splitting is proportional to the square of the magnetic field and can be understood by considering the corresponding eigenvectors at $q=0$. The first solution, $\tau_{+}^{-1}(q=0)$, is field independent, as it represents uniform tilt fluctuations in a plane perpendicular to the magnetic field. This mode is polar and the corresponding polarization is fluctuating along the direction of the magnetic field. The relaxation rate of the second mode $\tau_{-}^{-1}(q$ $=0)$ is field dependent and decreases with increasing field. This mode represents the tilt fluctuations along the direction of the magnetic field and the corresponding polarization fluctuates in a direction perpendicular to the magnetic field. It should be noted that the lower branch of excitations is critical and the lowest-lying mode on the dispersion condenses at the $\lambda$ line. For fields lower than the Lifshitz field, this happens at finite critical wave vector $q_{c}$, whereas at and beyond the Lifshitz field the mode condenses at $q=0$, thus inducing a phase transition into a homogeneous and unwound tilted smectic phase. 


\section{Phason dynamics for $T \ll T_{\lambda}$}

The general description of the spectrum of elementary excitations of a magnetic-field distorted smectic- $C^{*}$ phase just below the smectic- $A$ phase is complicated, as one has to consider the solitonlike nature of the amplitude and phase modes. However, it is well known that for zero external field the soft mode of the smectic- $A$ phase splits into amplitude and phase fluctuations in the unperturbed chiral smectic- $C^{*}$ phase [12]. The external magnetic field induces a band structure of both amplitude and phase fluctuations and induces band gaps in the originally gapless dispersion relation [6]. We can therefore expect four dispersion branches in the very vicinity of the $\lambda$ line, two of them representing amplitude and the other two phase fluctuations. However, KutnjakUrbanc [10] showed that the CAA approximation is valid practically throughout the temperature interval of the tilted smectic phase. An exception is a very narrow temperature interval just below the phase transition line, where the CAA breaks down. The width of this region is estimated to be 10 $\mathrm{mk}$ and is irrelevant for most experimental conditions. We shall therefore use CAA to describe our experimental results.

In the CAA (see Ref. [8]), one considers the phasedependent part of the free-energy density [ Eq. (6)], as the amplitude (i.e., the magnitude of the tilt angle) is set fixed and cannot fluctuate. The nonequilibrium phase of the order parameter is

$$
\Phi(z, t)=\Phi_{o}(z)+\Psi(z) e^{-1 / \tau(H)} .
$$

Here, $\Phi_{o}(z)$ is the static part of the phase profile that satisfies the sine-Gordon equation, $\Psi(z)$ is the eigenvector of the fluctuations, and $\tau^{-1}(H)$ is the corresponding relaxation rate. $\Psi(z)$ is a solution of the Lame's equation of order one and has a structure of a Bloch function $[8,13]$. The eigenfunctions of particular interest are those at $q=0$ because these contribute to the electro-optic response and give rise to strong light scattering. These eigenfunctions and their corresponding eigenvalues are

$$
\begin{gathered}
\Psi^{(1)}=\operatorname{cn}(u, k), \quad \tau_{-}^{-1}(q=0)=\frac{1-k^{2}}{k^{2}} \frac{\Delta \chi}{\gamma} H^{2}, \\
\Psi^{(2)}=\operatorname{sn}(u, k), \quad \tau_{+}^{-1}(q=0)=\frac{1}{k^{2}} \frac{\Delta \chi}{\gamma} H^{2} .
\end{gathered}
$$

Here $\operatorname{cn}(u, k)$ and $\operatorname{sn}(u, k)$ are the Jacobian cosine and sine amplitudes, respectively. The relaxation rate of the first mode (i.e., the acousticlike mode) decreases with increasing magnetic field and equals zero at the critical field. This mode is not present above the critical magnetic field. The relaxation rate of the second opticlike mode increases with increasing magnetic field and is stable for fields beyond the critical field. As a result, we have a magnetic-field-induced gap at $q=0$, which increases with the square of the field strength [8]. These modes are also polar and the polarization fluctuates in a direction perpendicular to the field for the acousticlike mode and in the direction of the magnetic field for the opticlike mode [8].

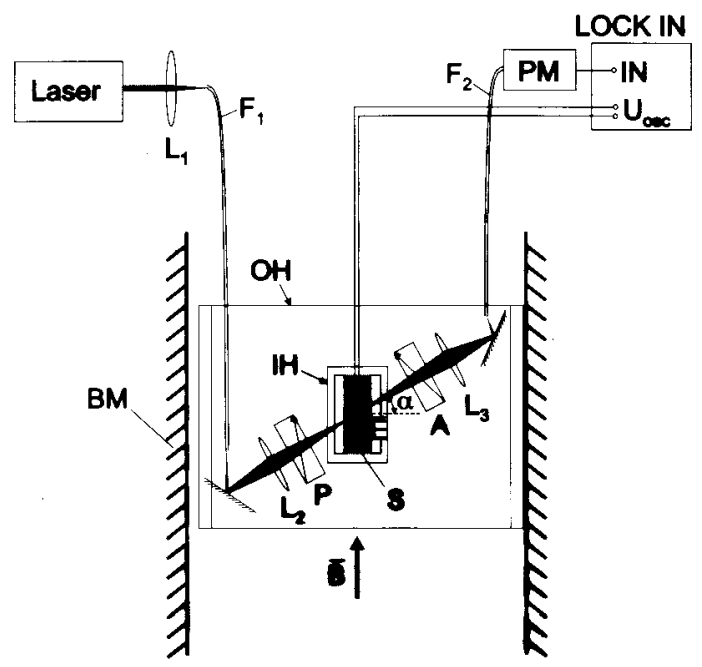

FIG. 3. Optical setup for the linear electro-optic response measurements of ferroelectric liquid crystal in external magnetic field. External magnetic field $\vec{B}$, smectic layer normal, and measuring electric field $\vec{E}$ are perpendicular to each other. Definitions: $L_{1}, L_{2}, L_{3}$-lenses; $P$, polarizer; $A$, analyzer; $S$, sample; IH, inner heater; $\mathrm{OH}$, outer heater; $F_{1}, F_{2}$-fibers; $\mathrm{PM}$, photomultiplier; $\mathrm{BM}$, Bitter magnet.

\section{EXPERIMENT}

The experiments were performed at the High Field Magnet Laboratory at the RIM, Nijmegen. In the experiment we have measured the linear electrooptic response and the autocorrelation function of the quasielastically scattered light in the smectic- $A$ and smectic- $C^{*}$ phases of a ferroelectric liquid crystal as a function of temperature and magnetic field.

The linear electro-optic experiment was performed in a miniaturized optical setup (Fig. 3) fitting into the 32-mm bore of a Bitter magnet (BM). A 20-mW He-Ne laser was focused into the optical fiber $F_{1}$, which guided the light into the setup placed in the center of the BM. The light transmitted through the sample $S$ placed between crossed polarizers was collected with a lens $L_{3}$ and focused into an optical fiber $F_{2}$. The angle $\alpha$ between the light direction and the optical axis of the sample was set to the half intensity of the transmitted light, thus yielding a maximum linear response for a homeotropically aligned sample. The fiber $F_{2}$ was connected to the photomultiplier PM which was connected to the lock-in amplifier. The lock-in amplifier measured the real (in-phase) and imaginary (out-of-phase) parts of the linear electro-optic response. It has been shown that this method is an optical analog of dielectric spectroscopy and one can detect polar eigenmodes of the system $[14,15]$.

In the experiment we have studied a mixture, consisting of $35 \%$ of pure chiral liquid crystal 4-(2'-methylbutyl)phenyl 4-n'-octylbiphenyl-4-carboxylate (CE-8 or 8SI) and 65\% of racemic liquid crystal CE-8R, that was studied by us previously $[6,8]$. Homeotropically aligned samples of $120-\mu \mathrm{m}$ thickness were prepared between two clean glass plates treated with dimethyloctadecyl-3-(trimethoxysilyl) propylammonium chloride (DMOAP). Two 100- $\mu$ m-thick copper wires were put parallel inside the cell. The distance between the wires was $1.5 \mathrm{~mm}$ to ensure a homogenous electric field at the measuring point. Special attention was paid to the 
temperature stabilization of the sample. The sample was placed in a double stage temperature controlled oven. The temperature stability was better than $2 \mathrm{mK}$. The measurements were performed by slowly decreasing the temperature in steps of $5-10 \mathrm{mK}$ at a constant magnetic field.

As the directions of the external magnetic field, smectic layer normal, and the measuring electric field were perpendicular to each other, the electric field couples in this geometry to the acousticlike phason mode in the distorted smectic$C^{*}$ phase, whereas in the smectic- $A$ phase it couples to the soft mode. In the smectic- $A$ as well in the smectic- $C^{*}$ phase we have indeed observed a single-mode response with a Debye-like spectrum. This spectrum is a result of a linear electro-optic response that is analogous to the dielectric response $\epsilon=\Delta \epsilon /(1+i 2 \pi \nu \tau)$, where $\Delta \epsilon=\epsilon(0)-\epsilon_{\infty}$ is the dielectric strength, $\tau^{-1}$ is the relaxation rate of the mode, and $\nu$ is the frequency of the measuring electric field. In the smectic- $A$ phase we attribute this relaxation to the soft mode and in the smectic- $C^{*}$ phase to the acousticlike phase mode.

The light scattering experiment was performed in an improved quasielastic light scattering spectrometer, fitting into the $60-\mathrm{mm}$ bore of a Bitter magnet [6,8]. The setup was double-temperature controlled thus yielding a milli-Kelvin temperature resolution. A He-Ne ordinary polarized laser beam was slightly focused to a $50-\mu \mathrm{m}$ spot, illuminating a $120-\mu \mathrm{m}$-thick homeotropically aligned liquid crystal sample placed in the center of the magnet. The scattered extraordinary light was collected into a multimode fiber and fed into a photomultiplier, placed far away from the magnet. The axis of the light-collecting optics was positioned at an angle of $\approx 1^{\circ}$ from the direction of the incoming light. In this geometry the light scattering originates from the modes with a wave vector along the helical axis. The heterodyne detected autocorrelation function could be fitted to a single exponential in the smectic- $A$ phase and to a double exponential in the tilted smectic phases. The light scattering experiment was much more susceptible to mechanical noise and thus inferior to the linear electro-optic response. The two sets of the experiment on different samples, however, gave nearly identical phase diagrams and order parameter dynamics.

\section{RESULTS AND DISCUSSION}

Figure 4 shows the temperature dependence of the relaxation rate $\tau^{-1}$ and the inverse value of the dielectric strength $\Delta \epsilon^{-1}$ at three different strengths of the magnetic field. For all fields and temperatures we have observed a single relaxation. In the smectic- $A$ phase this relaxation corresponds to the soft mode, whereas in the magnetically deformed smectic- $C^{*}$ phase it corresponds to the acousticlike phason [Eq. (14a)].

When the magnetic field strength was below $6 \mathrm{~T}$, we have observed the transition from the smectic- $A$ to the distorted smectic- $C^{*}$ phase [Fig. 4(a)]. In the smectic- $A$ phase both $\tau^{-1}$ and $\Delta \epsilon^{-1}$ decrease linearly with decreasing temperature. The transition temperature was determined by linear extrapolation of $\tau^{-1}$ and $\Delta \epsilon^{-1}$ in the smectic- $A$ phase to zero. Using this extrapolation, the phase transition point could be located with an accuracy of $10 \mathrm{mK}$. At the phase transition we have also observed a significant break in the slopes of the temperature dependencies of both $\tau^{-1}$ and

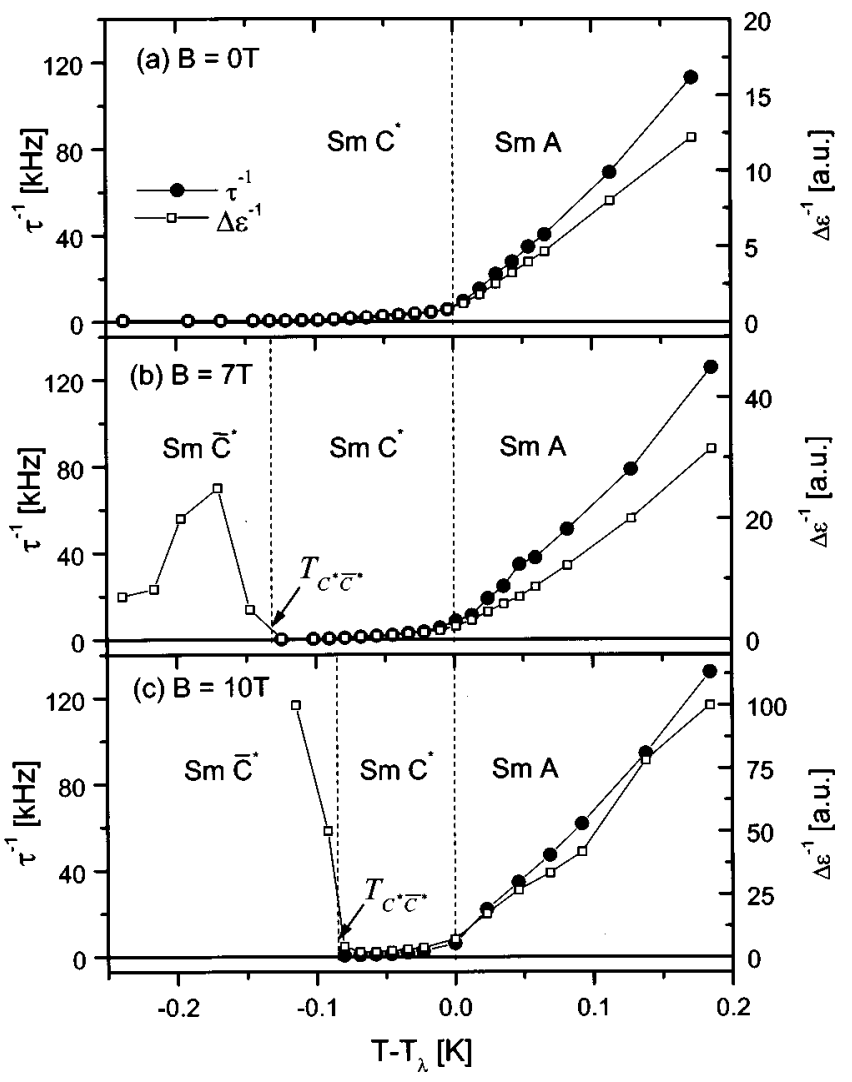

FIG. 4. Temperature dependence of the relaxation rate $\tau^{-1}$ and of the inverse value of the dielectric strength $\Delta \epsilon^{-1}$ of the soft mode in the smectic- $A$ phase and of the phase mode in the smectic- $C^{*}$ phase in a transverse magnetic field at $B=0 \mathrm{~T}$ (a), $B=7 \mathrm{~T}$ (b), and $B=10 \mathrm{~T}$ (c) as determined by the electro-optic response. Phase transition temperature to the unwound smectic- $\bar{C}^{*}$ phase is denoted by $T_{C * \bar{C}^{*}}$.

$\Delta \epsilon^{-1}$. In the magnetically deformed smectic- $C^{*}$ phase we have observed only one phase mode, i.e., the acousticlike phase mode, which is easily identified due to its low relaxation rate. The amplitude mode, which has a much lower intensity, could not be observed in linear electro-optic response experiments, but could be observed in the quasielastic light scattering experiments shown in Fig. 5. The temperature dependence of the relaxation rate of the acoustic phason is strongly correlated to the temperature dependence of the period of the helix. First it decreases, then it reaches a minimum value about $150 \mathrm{mK}$ below $T_{\lambda}$ and finally it gradually increases.

At a magnetic field strength of $6 T$ and above we have observed a very interesting behavior of the temperature dependence of the relaxation rate and dielectric strength, which is illustrated in Fig. 4(b). After slowing down in the smectic- $A$ phase, the relaxation rate further decreases in the modulated smectic- $C^{*}$. However, approximately $100 \mathrm{mK}$ below the $\lambda$ line, the electro-optic signal suddenly disappears and we observe only a very weak, spurious signal. This is a clear indication of the transition into the unwound smectic$\bar{C}^{*}$ phase with decreasing temperature. At this field strength we therefore first reach the region of the reentrant modulated smectic- $C^{*}$, which is also observed for higher fields. As another example, we show in Fig. 4(c) the temperature dependence of the relaxation rate and inverse dielectric strength 


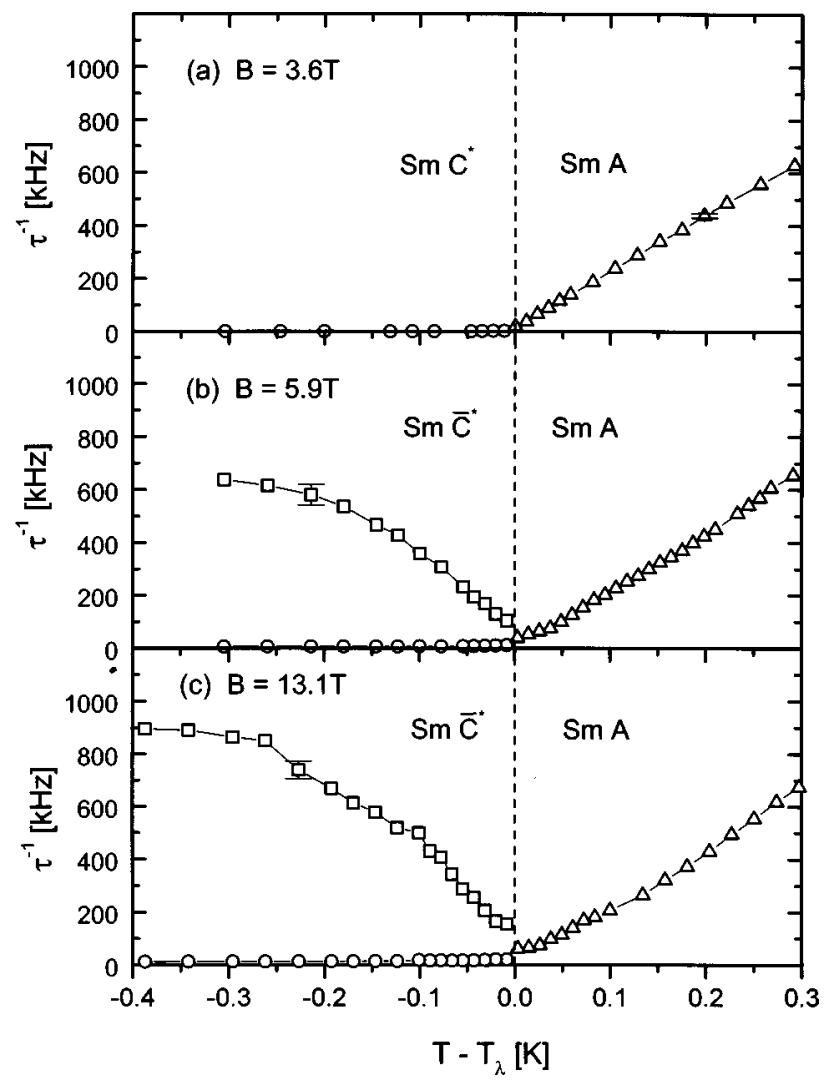

FIG. 5. Order parameter relaxation rates as determined by the quasielastic light scattering in a transverse magnetic field. For low field (a), the amplitudon cannot be resolved due to the large intensity of the low-frequency signal. For high field (b) and (c), this acousticlike phason is replaced by a weaker signal from the opticlike phason $(\bigcirc)$, which has a much lower intensity. This allows for the unambiguous determination of the amplitudon fluctuations ( $\square$ ).

at $10 \mathrm{~T}$. One can note, that the width of the reentrant phase is narrower but clearly detectable. This phase sequence smectic- $A$-smectic- $C^{*}$-smectic- $\bar{C}^{*}$ can be observed up to the highest field of $17 \mathrm{~T}$ that was used in our experiments.

The corresponding spectra of the linear electro-optic response are shown in Fig. 6 for $B=7.5 \mathrm{~T}$. We show only the most interesting spectra inside the modulated, magnetically deformed smectic- $C^{*}$ phase. One can clearly see that by decreasing the temperature, the strength of the signal increases, whereas the corresponding relaxation rate decreases (see the arrows in Fig. 6). The relaxation rate gets as low as several tens of hertz close to the transition into the unwound phase at $T_{C * \bar{C} *}$ (see Fig. 4). The phase transition is here of first order, as it is accompanied by a discontinuity of the relaxation rate and consequently also a discontinuity of the length of the helical modulation. Finally, in the unwound smectic- $\bar{C}^{*}$ phase there is practically no detectable signal, as shown in Fig. 6(d).

The temperature dependence of the order parameter relaxation rates, as determined from the quasielastic light scattering data are shown in Fig. 5 for three different strengths of the magnetic field. The wave vector of the excitations was chosen along the $z$ direction, $\vec{q}=(0,0,1.2) \mu \mathrm{m}^{-1}$. The data are compatible with the linear electro-optic response, except in the tilted phases. Here, we clearly observe the emergence of the amplitudon excitations, as shown in Figs. 5(b) and

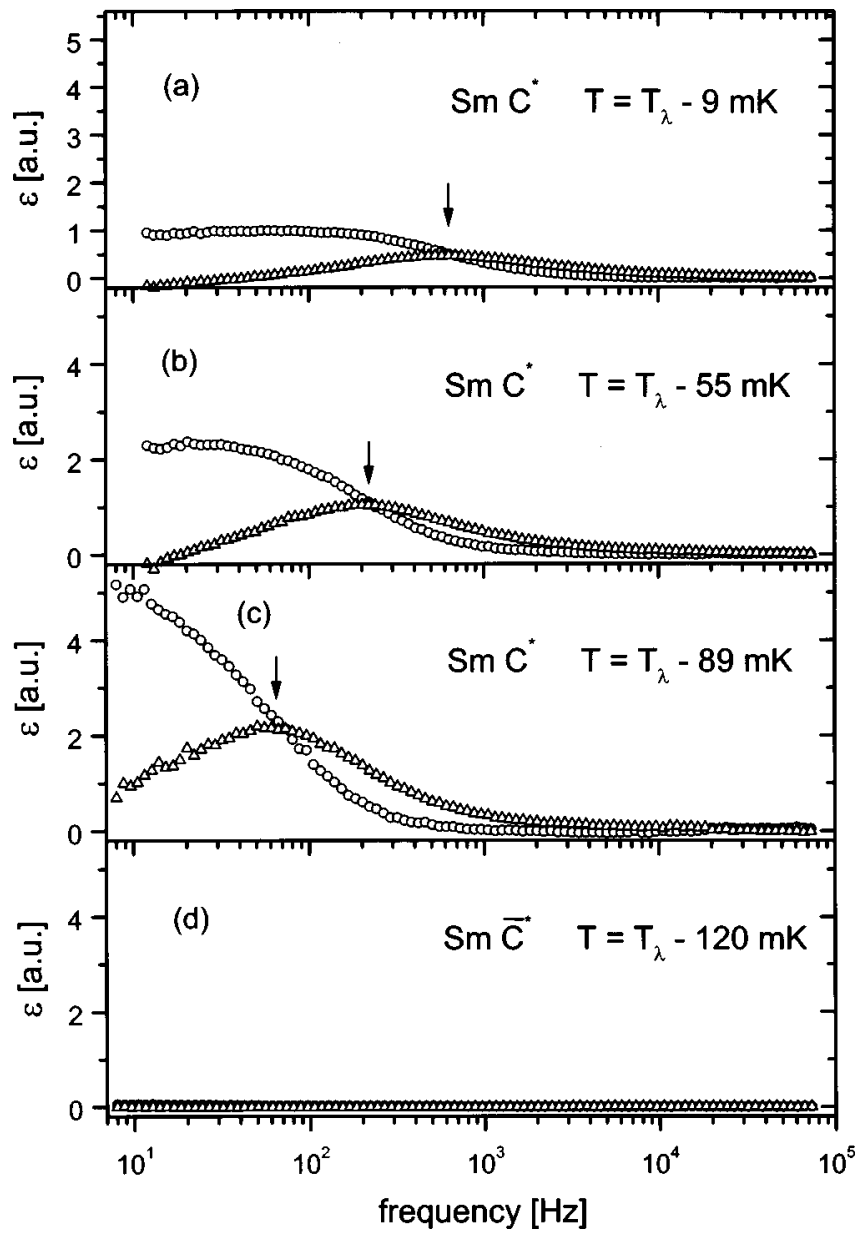

FIG. 6. Temperature and frequency dependence of the real $(\bigcirc)$ and imaginary $(\triangle)$ parts of the linear electro-optic response in the vicinity of the smectic- $C^{*}$ to the unwound smectic- $\bar{C}^{*}$ phase transition at $B=7.5 \mathrm{~T}$. Below the smectic- $A \rightarrow$ smectic- $C^{*}$ phase transition temperature $T_{\lambda}$ the static response of the phase mode increases and its relaxation frequency $\nu$ decreases. In the smectic- $\bar{C}^{*}$ phase the signal is very weak and we cannot determine the relaxation frequency. The arrows indicate the value of the relaxation frequency, which decreases when approaching the unwound phase.

5(c). Unfortunately, we could not resolve with sufficient accuracy both phason modes for low magnetic fields, as the splitting between these modes is only of the order of a $\mathrm{kHz}$. The same argument applies to the observed single amplitudon branch, because the splitting is many orders of magnitude smaller than the relaxation rates of the two amplitudons themselves.

From a large number of linear electro-optic response and light scattering experiments, performed by temperature sweeps at given $H$, we were able to construct the $(H, T)$ phase diagram of a $35 \%-65 \%$ mixture of pure and racemic CE-8 in a transverse magnetic field with a nearly milli-Kelvin resolution, which is shown in Fig. 7. All measurements have been done in one day and the results have been corrected for the observed shift of $T_{c}$ due to the sample aging $(\approx 7 \mathrm{mK} / \mathrm{h})$. One should note that the full temperature scale of the phase diagram is only $900 \mathrm{mK}$.

The $\lambda$ line is slowly increasing with magnetic field as is predicted for a material with positive diamagnetic anisotropy [2]. On the other hand, the line of the critical magnetic field 


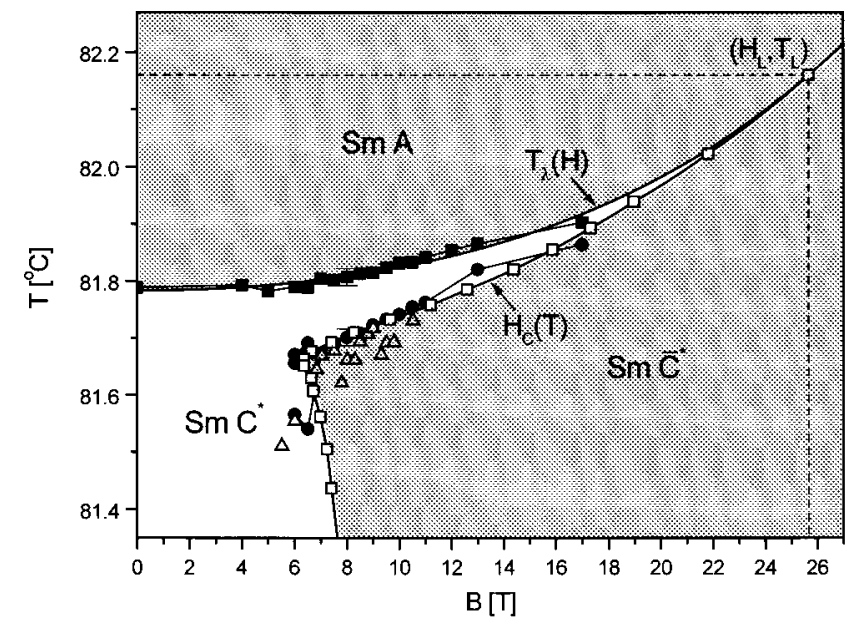

FIG. 7. Experimental $(B, T)$ phase diagram of a $35 \%-65 \%$ ferroelectric mixture of chiral and racemic CE8 in an external magnetic field as determined by the linear electro-optic response (filled squares and dots) and quasielastic light scattering (empty triangles). The upper solid line is the best fit to Eq. (3) with $\Delta T=0.13 \mathrm{~K}$ and $H_{L}=25 \mathrm{~T}$. The line of the critical magnetic field $H_{c}(T)$ was determined by the help of Eq. (15) which relates the phason relaxation rate to the critical magnetic field. Filled dots $(*)$ represent directly measured critical magnetic field. Empty squares $(\square)$ represent values obtained from Eq. (15) with measured temperature dependence $\tau^{-1}(T)$ at $B=0$ and $\Delta \chi / \gamma=30(1 \pm 0.1) \mathrm{T}^{-2} \mathrm{~s}^{-1}$. The solid line that connects the squares is a guide to the eyes. The Lifshitz field for this line is $H_{L}=25(1 \pm 0.1) \mathrm{T}$.

$H_{c}(T)$ is strongly temperature dependent. At low temperatures, i.e., $\approx 200 \mathrm{mK}$ below $T_{\lambda}$, the critical magnetic field for the unwinding of the helix is $\approx 7 \mathrm{~T}$ (see Fig. 7). This critical field increases gradually to $8 \mathrm{~T}$ with decreasing temperature [6]. However, approximately $150 \mathrm{mK}$ below $T_{\lambda}$, the critical magnetic field increases strongly with increasing temperature and we enter into the region of the reentrant modulated phase, first discovered in the experiments on DOBAMBC [3]. The reason for this strong increase of the critical magnetic field is the decrease of the helical period, as we approach the $T_{\lambda}$ line. This anomaly is common to most liquid crystal ferroelectric materials that have a second order smectic- $A-$ smectic- $C^{*}$ transition. According to Eq. (7), the critical field is inversely proportional to the helical period and therefore increases with decreasing helical period. In all our experiments, we have not observed any direct transition from the smectic- $A$ to smectic- $\bar{C}^{*}$ up to the highest measuring field of $17 \mathrm{~T}$. The temperature interval of the reentrant phase is rather small $(\approx 0.1 \mathrm{~K}$ at $\approx 8 \mathrm{~T})$ and decreases with increasing magnetic field. At very high magnetic fields (15 $\mathrm{T})$ the width of this reentrant phase is only around $40 \mathrm{mK}$. It seems that the width indeed goes to zero for stronger magnetic fields as predicted by theory [2].

As we have not succeeded to observe the Lifshitz point directly due to the enormously high value of this field, we have analyzed whether we can extrapolate the line of the critical field $H_{c}(T)$ to the range above $20 \mathrm{~T}$. As we know that the CAA approximation is valid nearly to the $10 \mathrm{mK}$ region close to the Lifshitz point, we extrapolate the line of the critical field within the CAA approximation. Here, the critical magnetic field is given by Eq. (7) i.e., $H_{c}$

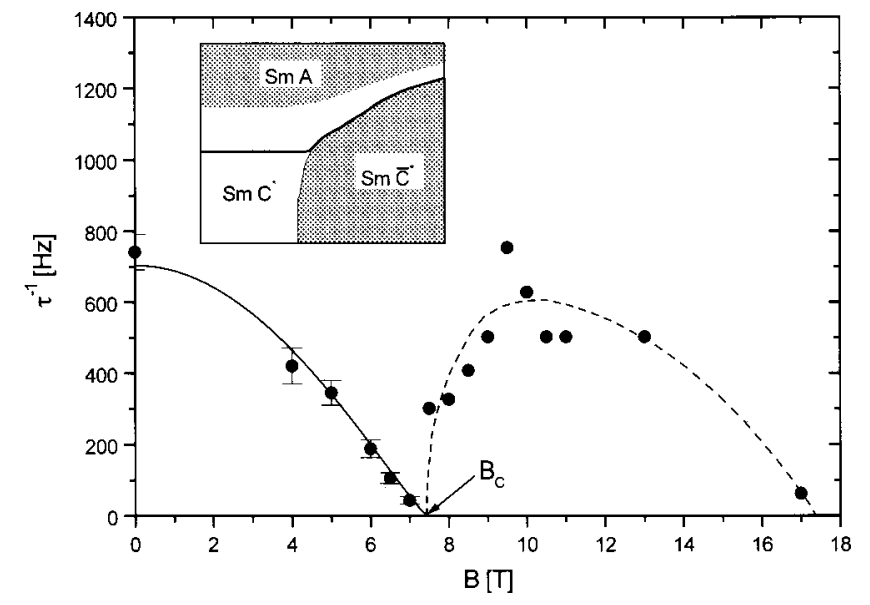

FIG. 8. The relaxation rate $\tau^{-1}$ of the acousticlike mode in the modulated smectic- $C^{*}$ phase as a function of the external magnetic field. The solid line represents the best fit to Eq. (15) with the critical field $B_{c}=7.45 \mathrm{~T}$ and $\Delta \chi / \gamma=31(1 \pm 0.1) \mathrm{T}^{-2} \mathrm{~s}^{-1}$. The dashed line above $B_{c}$ is a guide to the eyes. The inset shows the trajectory in the $(B, T)$ phase diagram, where the data points have been taken.

$=\left(\pi^{2} / p_{0}\right) \sqrt{K_{33} / \Delta \chi}$. Since the period of the unperturbed helix $p_{0}$ is directly related to the phason relaxation rate of the unperturbed helix, we can express the temperature dependence of the critical magnetic field $H_{c}$ with the phason relaxation rate $\tau^{-1}$

$$
\tau^{-1}=\frac{K_{33}}{\gamma}\left(\frac{2 \pi}{p_{0}}\right)^{2}, \quad H_{c}^{2}(T)=\frac{\pi^{2}}{4} \frac{\gamma}{\Delta \chi} \tau^{-1}(T) .
$$

The temperature dependence of the relaxation rate at $H=0$ has been measured directly, as well as the critical field in a temperature interval between 50 and $250 \mathrm{mK}$ below $T_{\lambda}$. Following Eq. (15) we can determine from these measurements (16 data points) the value of $\Delta \chi / \gamma=30$ (1 $\pm 0.1) \mathrm{T}^{-2} \mathrm{~s}^{-1}$. This allows us to extrapolate the line of the critical magnetic field beyond $13 \mathrm{~T}$ and up to the Lifshitz field, as shown in Fig. 7. We see that the agreement between the direct measurement of the critical magnetic field (filled circles) and the extrapolated critical magnetic field from the relaxation rate data (open squares, connected with a solid line) is rather good throughout the whole phase diagram. The triple point, as determined from this extrapolation is located at $25 \mathrm{~T}$. On the other hand, the field dependence of the $\lambda$ line (see Fig. 7) shows a clear increase as we approach the vicinity of the triple point. This line was fitted to Eq. (3) and is shown by the upper solid line in Fig. 7. In this fit we had to fix the value of the Lifshitz field to $25 \mathrm{~T}$, as the shape of the $\lambda$ line is quite insensitive to the magnitude of this parameter From the fit, we obtain $\Delta T \approx 0.13 \mathrm{~K}$. This is a reasonable value for the difference between the transition temperatures of pure and chiral liquid crystals and is close to the estimated value of $\Delta T \approx 0.08 \mathrm{~K}$ for $\mathrm{CE}-8$. One should, however, note that the accuracy of this line is low, due to the drift of the phase transition temperature.

The question of whether this triple point is indeed a Lifshitz point can be resolved by considering the character of the line of the critical magnetic field $H_{c}(T)$. As pointed out by Michelson, far below the $\lambda$ line, the unwinding transition 
is continuous and changes into first order upon approaching the tricritical point. Then, close to the Lifshitz point, the phase transition becomes again of second order.

Figure 8 shows the relaxation rate of the acoustic phason, measured throughout the trajectory of the $(H, T)$ phase diagram, which is shown by the solid line in the inset to Fig. 8 . When approaching the critical magnetic field for unwinding of the helical structure, the relaxation rate decreases continuously to zero. The solid line represents the best fit to Eq. (14a) with $\Delta \chi / \gamma=31(1 \pm 0.1) \mathrm{T}^{-2} \mathrm{~s}^{-1}$ and one can see that the agreement with theory is good. However, by continuing along the "edge of the horn" of the reentrant phase, the relaxation rate at the phase transition increases considerably. This suggests the existence of a discontinuous phase boundary, which becomes again continuous, as we approach the triple point (see the last point at $17 \mathrm{~T}$ ). This is an indication that the triple point which we have observed in the $(H, T)$ phase diagram of a ferroelectric liquid crystal in an external magnetic field is indeed a Lifshitz point.

\section{CONCLUSIONS}

Using quasielastic light scattering and linear electro-optic response measurements we have studied the order parameter dynamics near the Lifshitz point in the $(H, T)$ phase diagram of a ferroelectric liquid crystal in a transverse magnetic field. The extrapolated Lifshitz field for a 35\%-65\% mixture of a chiral and racemic CE- 8 is $25 \mathrm{~T}$, which explains why the Lifshitz point could not be observed in purely chiral DOBAMBC many years ago. We have confirmed without doubt the presence of the reentrant helicoidaly modulated smectic- $C^{*}$ phase. The width of this phase is of the order of $100 \mathrm{mK}$ at $6 \mathrm{~T}$ and decreases gradually as we approach the Lifshitz point.
[1] R. M. Homreich, M. Luban, and S. Shtrikman, Phys. Rev. Lett. 35, 1678 (1975).

[2] A. Michelson, Phys. Rev. B 16, 585 (1977).

[3] I. Muševič, B. Žekšs, R. Blinc, Th. Rasing, and P. Wyder, Phys. Rev. Lett. 48, 192 (1982).

[4] A. Seppen, Ph.D. thesis, University of Nijmegen, 1987; see also R. Blinc, I. Muševič, B. Žekšs, and A. Seppen, Phys. Scr. T35, 38 (1991).

[5] Z. H. Wang, Z. M. Sun, and D. Feng, Europhys. Lett. 14, 785 (1991).

[6] I. Muševič, B. Z̆ekš, R. Blinc, H. A. Wierenga, and Th. Rasing, Phys. Rev. Lett. 68, 1850 (1992).

[7] I. Muševič, B. Žekš, R. Blinc, M. M. Wittebrood, and Th. Rasing, Physica B 211, 331 (1995).
[8] I. Muševičc, B. Žekšs, R. Blinc, and Th. Rasing, Phys. Rev. B 49, 9299 (1994).

[9] L. Benguigui, and A. E. Jacobs, Phys. Rev. E 49, 4221 (1994).

[10] B. Urbanc-Kutnjak, Ph.D. thesis, University of Ljubljana, 1993.

[11] P. Byrd and M. D. Friedman, Handbook of Elliptic Integrals for Engineers and Physicists (Springer-Verlag, Berlin, 1954).

[12] I. Mušsevič , R. Blinc, B. Zekšs, C. Filipič, M. Copič , A. Seppen, P. Wyder, and A. Levanyuk, Phys. Rev. Lett. 60, 1530 (1988).

[13] B. Sutherland, Phys. Rev. A 8, 2514 (1973).

[14] W. Kuczynski, J. Hoffman, and J. Malecki, Ferroelectrics 150, 279 (1993).

[15] M. Škarabot, I. Muševič, and R. Blinc, Phys. Rev. E 57, 6725 (1998). 